\title{
Une base de données pour l'enseignement des langues régionales, de leurs cultures et territoires
}

Pierre Boutan et Micheline Cellier

\section{(2) OpenEdition}

Journals

Édition électronique

URL : http://journals.openedition.org/trema/920

DOI : 10.4000/trema.920

ISSN : 2107-0997

Éditeur

Faculté d'Éducation de l'université de Montpellier

Édition imprimée

Date de publication : 1 septembre 2009

Pagination : 17 - 24

ISSN : 1167-315X

Référence électronique

Pierre Boutan et Micheline Cellier, «Une base de données pour l'enseignement des langues régionales, de leurs cultures et territoires », Tréma [En ligne], 31 | 2009, mis en ligne le 01 septembre 2011,

consulté le 21 avril 2019. URL : http://journals.openedition.org/trema/920 ; DOI : 10.4000/trema.920

Ce document a été généré automatiquement le 21 avril 2019

Trema 


\title{
Une base de données pour l'enseignement des langues régionales, de leurs cultures et territoires
}

\author{
Pierre Boutan et Micheline Cellier
}

1 La recherche «Enseigner la région» dirigée par Pierre BOUTAN à l'IUFM de Montpellier ${ }^{1}$ a montré l'intérêt, au moment où s'élargissent les horizons institutionnels au - delà de l'habituel national, de s'appuyer sur la dimension du monde proche, local et régional, directement vécu par les élèves.

2 Dès 2003, il est apparu nécessaire de continuer cette recherche en lui donnant

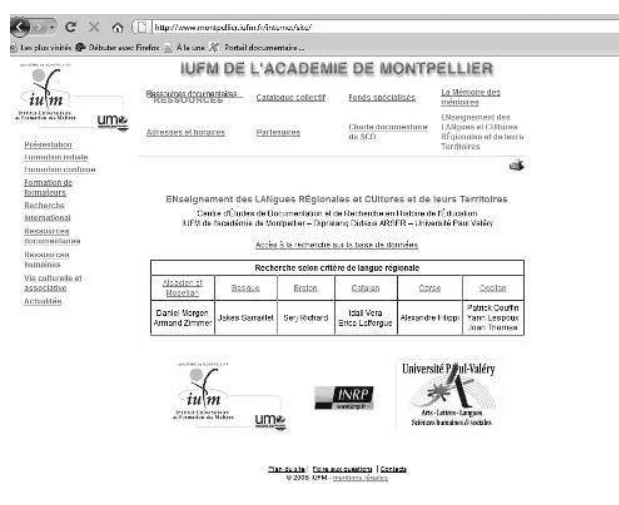
un objectif très ciblé, conforté par la généralisation au primaire de l'apprentissage des langues vivantes et régionales. Il devenait important de recenser les outils pédagogiques et de constituer une base de données sur l'enseignement des langues et cultures régionales parlées en France, pensées dans leurs temps et leurs espaces de référence. La consultation des sites sur Internet qui leur sont dédiés montrait qu'il n'existait pas d'instrument de travail de cette nature.

Les produits proposés étaient soit des bibliographies circonscrites à une langue, sans dimension critique, soit des outils d'apprentissage, soit des informations générales. À partir de ce constat, il s'est agi de constituer l'ensemble général et critique qui manquait encore pour inventorier les documents permettant l'enseignement des langues régionales, de leurs cultures et de leurs territoires. Cette vaste base de données a trait pour l'instant aux langues parlées dans l'Hexagone et en Corse; elle prend en considération plusieurs médias : manuels, dictionnaires, atlas, albums, CD, DVD, films vidéos ... Pour en permettre la consultation et la diffusion, un site Internet a été créé. 


\section{Constitution de la base et du site}

La recherche ${ }^{2}$ a été lancée en 2004, impliquant l'IUFM de Montpellier et l'Université Paul Valéry - Montpellier III', l'INRP puis l'UMR Éducation \& Politique de l'Université Lumière Lyon $\mathrm{II}^{4}$ en 2007 ; à partir de 2008, un rapprochement s'est opéré avec le groupe de recherche INRP - ICAR.

5 La base de données créée n'est ni générale, ni exhaustive. La perspective d'ensemble est pédagogique et didactique: l'objectif est de répertorier en priorité les ouvrages habituellement utilisés dans les classes et de faire ainsi un état des lieux des objets permettant d'enseigner les langues régionales. Le recensement, dès lors, ne peut être assuré que par des spécialistes de la langue traitée et ayant une bonne connaissance du terrain ; ces rédacteurs régionaux ${ }^{5}$, répartis sur le territoire, sont professeurs, chargés de mission ou Inspecteurs Pédagogiques Régionaux en charge des langues régionales. Les notices présentées correspondent donc à une sélection opérée par des experts.

$\mathrm{BCDI}$, le logiciel de saisie choisi, est très répandu dans les institutions scolaires, ce qui facilite la coopération des services de documentation de tous les IUFM, CDDP, CRDP et organismes régionaux particuliers ${ }^{6}$ proches des rédacteurs qui sollicitent également d'autres structures, universitaires ou spécialisés ${ }^{7}$, en particulier le CEDHRE ${ }^{8}$.

7 La liste des langues retenues est issue de celle que fournit l'Éducation nationale pour ce qui est des langues enseignées dans le second degré: basque, breton, catalan, corse, langues régionales d'Alsace et de Moselle, occitan. Seuls les niveaux d'enseignement primaire et secondaire ont été retenus. Ultérieurement, on pourra envisager une extension à d'autres langues régionales territorialisées, un élargissement au niveau universitaire, et le traitement de documents relevant de l'histoire de ces enseignements.

8 La compilation de ces outils pédagogiques devant aboutir obligatoirement à sa large diffusion, via Internet, la base est en accès libre à partir du lien ${ }^{9}$ : http:// www.montpellier.iufm.fr/internet/site/

On peut consulter la totalité de la base, toutes langues confondues, ou un seul domaine linguistique sur lequel il suffit de cliquer. Actuellement, les bases, d'ampleur différente, contiennent près d'un millier de notices pour les langues d'Alsace et de Moselle et le breton, 600 pour l'occitan, 500 pour le basque. Seules les bases catalane et corse sont peu pourvues - pour des raisons tenant essentiellement à la difficulté de trouver des collecteurs spécialistes compétents et correspondant aux caractéristiques administratives permettant des attributions en moyens horaires. Elles sont tenues à jour, au fur et à mesure des saisies.

\section{Recherches sur la base de données}

La base est constituée de fiches signalétiques comportant des renseignements généraux (titre, date, auteur ... etc.) mais aussi une notice descriptive à dimension critique et pédagogique. Ces notices renvoient aux didactiques des disciplines savantes et scolaires (linguistique, psycho et sociolinguistique, littérature, histoire, géographie, et, plus accessoirement, SVT, mathématiques) et concernent les niveaux de l'école, du collège et du lycée. Les ouvrages peuvent être à destination des élèves, comme dans le cas des 
manuels, ou des enseignants pour les guides pédagogiques, par exemple, ou des deux. Voici un exemple de fiche, telle qu'elle se présente en consultation.

\begin{tabular}{l} 
Fiche complète \\
Titre : Bücherwurm Lesebuch 2. Jahr = Le livre de lecture 2ème année \\
Type Notice : Notice générale \\
Résumé : Manuel de lecture en allemand pour la deuxième année élémentaire, offrant un choix \\
renouvelé de textes variés, parfois un peu difficiles pour les élèves des classes bilingues à parité \\
horaire, mais souvent compatibles avec leur niveau en langue. Parmi le choix de lectures, classées \\
par thèmes, en étroite relation avec le Sprachbuch /manuel de langue, beaucoup de poèmes, de \\
contes et de textes à dramatiser (Hörspiel). Les relations entre lecture et production de textes, entre \\
lecture, activités et travaux, entre manuel de lecture et manuel de langue sont étroites et \\
intéressantes. CECRL : A2. \\
Descripteurs : allemand : langue \\
Mots clés : langue régionale d'Alsace et des pays mosellans \\
Natures : Manuel \\
Niveaux : CE1 \\
Publics : Apprenant \\
Disciplines N. : Allemand / lecture \\
Auteurs : Stangner, Isolde / Braun, Anne / Manthey, Heike \\
Editeurs : Klett \\
Date de parution : 2004 \\
Collection : Bücherwurm \\
Collation : 184 p. : ill. en coul. ; $20 \times 26 \mathrm{~cm}$ \\
Notes : Nouvelle édition. \\
Support N. : Livre Isbn : $3-12-270332-7$ \\
\hline
\end{tabular}

Plusieurs types de recherche sont possibles : par thèmes, par critères ou par thésaurus.

12 La recherche thématique (premier onglet proposé) peut être " rapide » ou " avancée ", comme il apparait dans la figure suivante. Dans le premier cas, sur la base complète, on peut chercher tous les documents traitant d'un sujet particulier, les contes, par exemple avec ou sans restrictions de recherche (date, support...). En l'état actuel de la base, si on clique sur «rechercher», 151 documents sont sélectionnés par le logiciel contenant le terme « conte » dans le titre, le résumé, le descripteur ou le mot - clé.

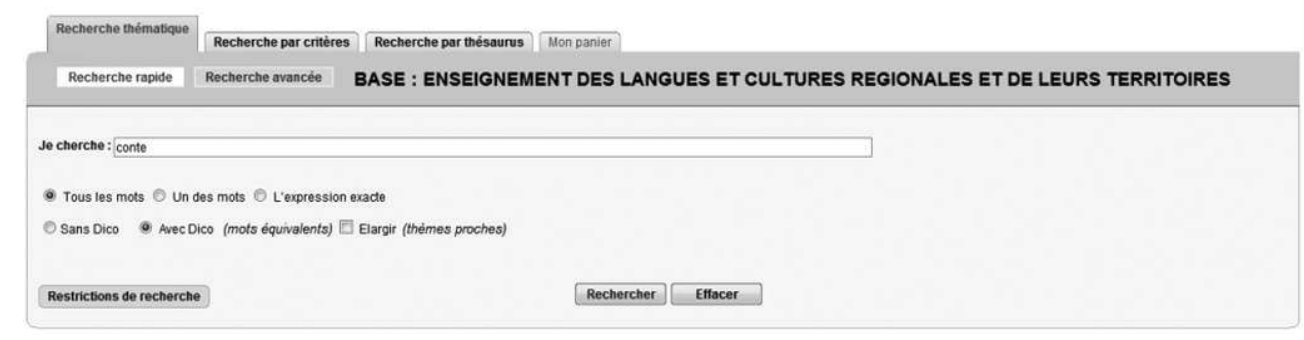

13 La recherche «avancée» permet de monter une équation à partir de plusieurs paramètres grâce à la fonction « et ", comme dans la plupart des moteurs de recherche. Ici, l'investigation peut être faite conjointement sur « conte » et " occitan », par exemple. 97 documents sont alors sélectionnés. 


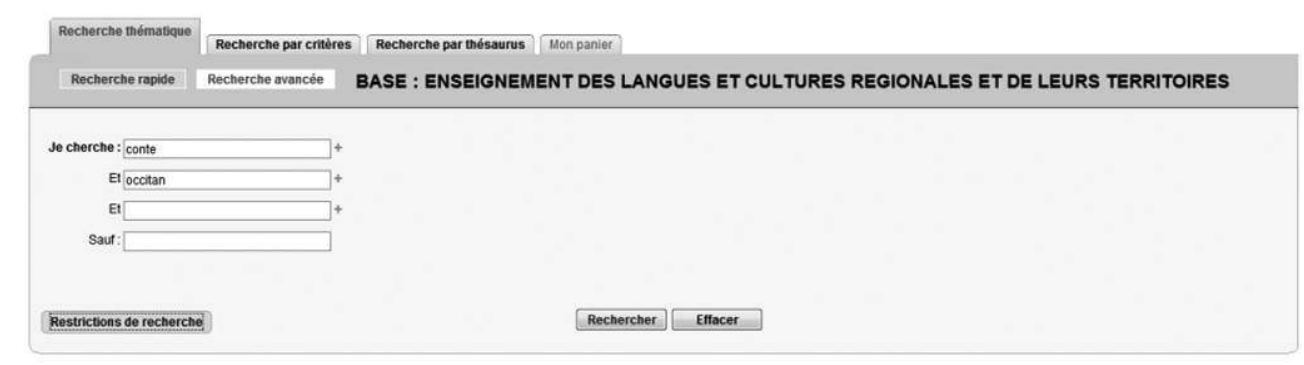

On peut, pour une recherche plus ciblée, choisir un seul critère à partir du second onglet et sélectionner au choix, le titre, l'auteur, l'éditeur, la collection, l'ISBN ou plus simplement utiliser la barre : "je cherche ", en mettant un ou plusieurs mots - en jouant sur les paramètres « tous les mots », « un seul des mots », «l'expression exacte ».

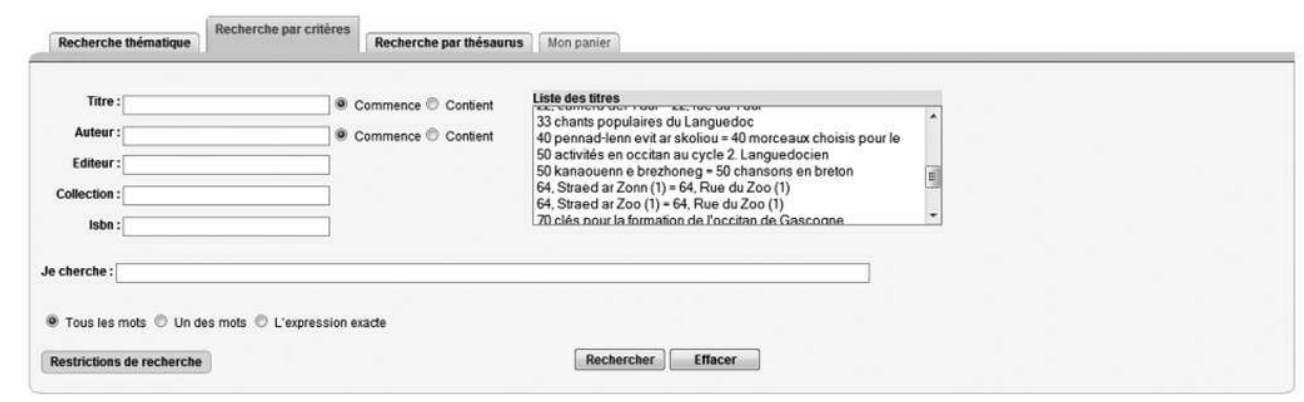

Le thésaurus (3e onglet) permet une recherche directe, à partir des mots descripteurs, indexés selon les normes en vigueur, connues des rédacteurs qui utilisent cette liste pré établie et fermée. La procédure est plus compliquée car elle suppose des connaissances de la part de la personne qui interroge la base : elle doit utiliser le bon descripteur. Mais tout un système de renvois aux mots génériques permet de ne pas laisser la recherche infructueuse. Par exemple, le mot descripteur «occitan» est employé pour «langue d'oc », «niçois: langue ", « limousin: langue », "provençal: langue». Si on tape "niçois ", la recherche est re - dirigée vers " occitan : langue » auquel sont associées toutes les fiches. Le descripteur le plus général subsumant l'ensemble est «langue régionale ».

16 Le logiciel offre donc une palette de recherches variée, permettant la sélection des données précises, en fonction des demandes particulières à chaque consultant qui pourrait ne s'intéresser qu'à un point précis - les manuels, la grammaire, le vocabulaire, la bande dessinée, la poésie, le théâtre, le conte ... - respectant ainsi la spécificité et la singularité de chaque requête.

\section{Intérêts de la base}

On sait à quel point la maîtrise de l'information devient une des clés de l'enseignement : rester isolé n'est plus possible. L'obligation de la transmission des connaissances rend incontournables tous les travaux bibliographiques.

La base constitue un inventaire utile pour tous les producteurs d'outils car elle valorise leurs travaux et rend lisible leur repérage sur le marché éditorial; elle est également précieuse pour les enseignants car elle leur permet de connaître le matériel relatif aux langues et cultures d'un domaine donné, de trouver des suggestions pédagogiques 
(notamment, en termes de support, le matériel audio - visuel - DVD, vidéos - étant aussi répertorié) et des outils de travail analysés.

Car, outre la quantité d'objets proposés dans certaines bases, il faut insister sur l'effort de qualité recherché dans les résumés. La fiche comporte, généralement, une partie descriptive du contenu - découpage et organisation des notions que l'objet se propose de faire acquérir, progression, conditions de mise en œuvre, séquences d'apprentissage ... qui donne une idée du projet des concepteurs, des moyens qu'ils utilisent pour amener à la construction des savoirs et des compétences. Mais l'analyse peut être critique : il s'agit d'évaluer la pertinence pédagogique d'un objet. Par exemple, une collection est identifiée comme problématique par les deux rédacteurs alsacien et breton. Le premier, dans le résumé de L'imagerie Français - Alsacien, signale des approximations inacceptables: « Imagier présentant plus de 500 mots en français avec leur traduction en dialecte. Mais, de fait, l'utilisation pédagogique de cet ouvrage fait problème à cause des variations inexpliquées de choix de graphie, tantôt dialectale, tantôt allemande (Exemple : on ne dit pas Apfelsine en alsacien, mais Orange ...), et à cause de plusieurs erreurs de choix sémantique et de transcription. Apparemment, l'imagier existe pour d'autres langues, le basque, le corse, le breton, l'occitan, le provençal... ». Le rédacteur breton s'étonne, lui aussi, de l'imprécision de la traduction dans L'imagerie Français - Breton: «A noter que les mots sont mentionnés en français à la forme indéfinie, alors que le breton les traduit à la forme définie : "An dañvad = le mouton, traduction = Un mouton ». » Les résumés rédigés par des spécialistes de la question, rendent compte des choix d'ouvrages en fonction des diversifications curriculaires (comme dans l'exemple sur le livre de lecture donné plus haut) et des compétences des élèves, y compris pour la littérature. Le rédacteur occitan détaille ainsi le résumé d'un roman, Josèp l'esclau $=$ Joseph l'esclave ${ }^{10}$ : "La vie au XIXe siècle, sur l'île de La Réunion, de Joseph, né esclave puis affranchi. Il s'agit d'un roman pour la jeunesse assez dense, ce qui fait qu'il sera plutôt à réserver à des élèves ayant un bon niveau de langue, Calandreta et section bilingue de primaire éventuellement, mais plus sûrement section bilingue de collège, d'autant plus que le thème est en relation directe avec le programme d'histoire. »

Depuis novembre 2008, les fiches produites cherchent à affiner l'analyse en définissant les critères d'évaluation des objets étudiés en regard des textes officiels, notamment le Cadre européen commun de référence pour les langues. Celles qui sont récemment créées (un travail est prévu en 2009 - 10 pour une évaluation rétroactive de celles qui ont été produites avant) comportent une indication du niveau visé dans l'échelle de compétences langagières du type :

A1 - niveau introductif de découverte, généralement demandé à l'école élémentaire

A2 - intermédiaire ou usuel qui correspond au 1er palier du collège (6e - 5e)

B1 - niveau seuil qui renvoie au 2e palier du collège $(4 e-3 e)$

B2 - niveau avancé pour le lycée.

L'utilisateur expérimenté entre dans le niveau C, jusqu'à la maîtrise totale de la langue qui correspond à $\mathrm{C} 2$.

Il est important de savoir à quel niveau de compétence langagière propose de former l'objet d'enseignement analysé mais la tâche est parfois difficile. Tous les ouvrages traités ne relèvent pas de ce type d'évaluation, les livres de littérature, ou les documents historiques ou géographiques en français, notamment. L'appréciation peut se compliquer dans quelques cas, en particulier pour les élèves bilingues pour lesquels les exigences sont 
décalées d'un cran vers le haut : A2 fin de l'école, B2 fin du collège, $\mathrm{C} 1$ en lycée. Le manuel d'allemand langue régionale de CE1 proposé plus haut en exemple s'adresse aux classes bilingues à parité horaire dont le niveau ne correspond pas au cursus normal : il serait plutôt de l'ordre de A2.

A rebours, l'échelle peut aussi être décalée vers le bas quand les élèves commencent l'apprentissage en 6e, sans initiation à l'école. Le niveau A2 est alors atteint seulement en fin de collège, pour ceux qui prennent l'option «langues régionales ». Mais cette échelle évaluative offre des repères nécessaires.

L'enseignement des langues régionales apparaît donc beaucoup plus diversifié que celui des langues vivantes qui démarre pour tous les enfants au même moment de la scolarité, d'où la nécessité de viser un niveau plutôt qu'une classe donnée.

Enfin, la base peut présenter un fort intérêt pour la recherche. Les chercheurs en didactique devraient en tirer profit car il faudrait passer du recensement à l'analyse pédagogique et didactique des objets. Le caractère général de la base qui inclut six langues régionales permet ainsi de croiser les travaux sur l'ensemble des domaines linguistiques, ce que la dispersion des données régionales dans chaque espace isolé rend difficile.

Ce premier recensement général qui donne une lisibilité nationale et internationale va autoriser des études comparatives portant sur bien des points, le champ de l'édition, par exemple : une exploration des bases spécifiques ou de l'ensemble fait apparaître la vitalité de certains éditeurs locaux et des CRDP - une bonne partie de la base bretonne est produite par TES ${ }^{11}$, par exemple - et permet de déterminer la proportion de productions originales dans la langue, par opposition aux traductions d'ouvrages existant déjà sur le marché national, la logique consistant parfois, surtout pour les disciplines non linguistiques, à reprendre des séries complètes en mathématiques ou en biologie.

31 L'analyse devrait se centrer essentiellement sur les didactiques pour dégager des entrées et des axes communs à l'apprentissage des langues régionales, les spécificités imposées à chacune d'elles par des contraintes particulières mais aussi le poids les dynamiques régionales - en termes d'éditions, certes, mais aussi d'impulsions institutionnelles ${ }^{12}$.

L'état des lieux donne des indications (d'autant plus intéressantes que la base est abondante) sur les types de pédagogie en présence dans les classes: il informe sur les supports privilégiés et renseigne de manière un peu précise sur ce qui est considéré comme devant être enseigné aux élèves - qui diffère, certes, de ce qui l'est réellement car il y a adaptation des outils et ajout de dispositifs complémentaires.

Il est évident, par exemple, que la littérature de jeunesse qui a fait une entrée massive à l'école à la suite d'Instructions officielles de 2002 est très employée, les textes les plus connus (patrimoniaux ou autres) étant traduits dans la plupart des langues régionales, comme Le Petit Prince traduit en basque, breton, francique rhénan, allemand ..., ce qui n'empêche pas la création d'œuvres spécifiques ou la valorisation d'auteurs ancrés dans la région, à des fins pédagogiques. Mais l'analyse qui est faite de ces ouvrages dans la base reste toujours en rapport avec l'apprentissage de la langue et culture régionale et diffère en ce point des notices trouvées habituellement dans les bibliothèques ${ }^{13}$. 
La base, outil inter - langues, ouvre les perspectives de confrontation entre les modes d'apprentissage de ces langues et cultures dont on sait combien, au - delà d'une étiquette commune, elles se trouvent dans des conditions très variées. La mise en œuvre de la base de données amène ainsi à renforcer les liens créés entre enseignants et chercheurs, tous ceux qui s'intéressent à ce domaine particulier de l'enseignement des langues.

Et s'il est vrai que, d'une manière générale, « l'identité éditoriale d'un pays, et donc aussi une part de son identité culturelle est portée par son système bibliographique, à la fois vitrine et mémoire patrimoniale ${ }^{14}$, il en est de même pour le domaine spécifique des langues régionales et les outils éducatifs permettant de les enseigner.

\section{BIBLIOGRAPHIE}

Alén GARABATO C. (Ed.). (2007). Les langues de France au XXIe siècle : vitalité sociolinguistique et dynamiques culturelles. Paris : L'Harmattan.

Alén GARABATO C. (2009). Langues minoritaires en quête de dignité. Le galicien en Espagne et l'occitan en France. Paris : L'Harmattan.

BOUTAN, P., MARTEL, P., ROQUES, G. (dir.) (2001). Enseigner la région. Actes du colloque international de Montpellier, 4 et 5 février 2000. Paris : L'Harmattan.

BOUTAN, $\mathrm{P}$ (2002). L'usage du manuel en question : une tradition en matière d'apprentissage des langues », Études de linguistique appliquée, $n^{\circ} 125$, janvier-mars, coord.VERDELHAN-BOURGADE M. «Les manuels scolaires, un discours didactique », pp. 11-24

CELLIER, M., TORREILLES, C., VERNY, M.J., (2004). Entre deux langues. Autobiographie et bilinguisme, textes commentés. Paris : éditions Adapt.

COSTA, J. (2008). Langues et cultures régionales, langues vivantes étrangères. Quels enjeux pour une convergence didactique? Cahiers de l'Acedle, 5(1), 109-128.

http ://acedle.u-strasbg.fr/article.php3?id_article=1019

Délégation générale à la langue française et aux langues de France : http:// www.dglf.culture.gouv.fr/

FREMONT, A. (1999). La région, espace vécu. Champs, Flammarion, 2nde éd.

LIEUTARD, H. et Verny, M.-J. (eds.) (2007). L'école française et les langues régionales-XIXe-XXe siècles. Montpellier : Presses Universitaires de la Méditerranée.

MARTEL P. (2003). Minority languages of metropolitan France : a long road, Acts of VIII international conference on minority languages, Santiago de Compostela, Xunta de Galicia, pp. 303-308.

\section{NOTES}

1. Recherche 1999 - 2002, IUFM de Montpellier - Didaxis Dipralang

2. Responsables : Carmen ALEN GARABATO, MCF sciences du langage, Dipralang, Pierre BOUTAN, MCF honoraire en sciences du langage, IUFM de Montpellier, Philippe MARTEL, chargé de recherche au CNRS, Redoc.

3. Laboratoire Dipralang - Didaxis et ARSER - EA 739 et Recherches en domaine occitan UMR CNRS ETOILL 
4. Recherche: «Le curriculum scolaire à l'épreuve du local et des populations à besoins spécifiques ».

5. Basque (Jakes SARRAILLET); Breton (Serj RICHARD) ; catalan (E. LAFFORGUE ; Idali VERA); Langues régionales d'Alsace et de Moselle (Daniel MORGEN, Armand ZIMMER); occitan (Joan THOMAS, Yann LESPOUX, Patrick COUFFIN) ; corse (Alexandre FILIPPI). Coordination nationale : Micheline CELLIER.

6. En particulier, le CAP'Oc (Centre d'Animation Pédagogique en Occitan) qui a mis sa base à notre disposition.

7. Comme le CIRDOC (Centre Inter - Régional pour le Développement de l'Occitan) pour la région Languedoc - Roussillon.

8. Centre d'Etudes de Documentation et de Recherche en Histoire de l'Education - IUFM de Montpellier

9. On peut suivre aussi le chemin suivant : à partir du portail de l'IUFM de Montpellier, prendre l'onglet «Recherche », à droite ; cliquer ensuite sur «CEDHRE » (3e ligne en haut), puis dans l'encadré sur «ENseignement des LANgues et CUltures REgionales et de leurs Territoires ». Ou bien, à partir du portail IUFM de Montpellier, prendre l'onglet "Ressources documentaires ", puis « ENseignement des LANgues et CUltures REgionales et de leurs Territoires ».

10. Gui VIALA, IEO, 2001.

11. TES, Ti - Embann ar Skolioù, centre régional multimédia de productions pédagogiques en langue bretonne qui diffuse des ouvrages gratuitement dans les établissements scolaires et en vend une partie dans les librairies.

12. Voir dans l'académie de Montpellier l'élaboration par le Recteur Christian NIQUE d'un «Plan de développement des langues régionales " et la rédaction collective, sous sa direction, de trois ouvrages sur l'occitan et le catalan, distribués dans tous les établissements : Précis d'occitan et de catalan (CRDP, 2006), Petite anthologie de la littérature occitane et catalane (2006), et Histoire du Languedoc Roussillon (2007).

13. Par exemple, voir l'analyse faite par le rédacteur occitan pour Titeuf ou le sens de la vie traduit en occitan (Titeuf, lo sens de la vida) en 2008 : «Les aventures du jeune Titeuf, à l'école, avec ses amis ou avec sa famille. Cette bande dessinée à succès bénéficie enfin d'une traduction en occitan. Comme cela semble être devenu la règle dans les traductions occitanes, les personnages s'expriment dans des dialectes différents (essentiellement en languedocien et gascon). Un bon moyen de travailler sur l'interdialectalité. CECRL : A2 à B2. »

14. BEAUDIQUEZ Marcelle, "Les services bibliographiques nationaux à l'aube du XXIe siècle : évolution et révolution », ICNBS, Copenhague, 25 - 27 novembre 1998, consultable à partir du lien : http://archive.ifla.org/VI/3/icnbs/beam.htm

\section{RÉSUMÉS}

Il est apparu nécessaire de recenser les outils pédagogiques permettant d'enseigner les langues régionales, leurs cultures et leurs territoires : c'est pourquoi ont été créés une base de données concernant les langues parlées dans l'espace métropolitain et un site Internet en permettant une large consultation. Cette base générale offre aux enseignants du primaire, du secondaire et du supérieur, un inventaire des productions pédagogiques dans un domaine linguistique et culturel 
donné et aux chercheurs l'outil nécessaire pour des études comparatives entre les différentes didactiques et approches de l'apprentissage des langues.

It appeared that it was necessary to make an inventory of the teaching aids to teach regional languages, their culture and their territories : that is why a data base about the spoken languages in the metropolitan area has been created as well as an Internet site for wide consultation. This general basis offers primary and secondary school teachers an inventory of pedagogical works in a given linguistic and cultural field and researchers the necessary tool for comparative studies between the different didactics and approaches of language learning.

\section{INDEX}

Mots-clés : base de données, internet, langue régionale, site

Keywords : database, regional language

\section{AUTEURS}

\section{PIERRE BOUTAN}

Maître de conférences honoraire, Didaxis Dipralang

\section{MICHELINE CELLIER}

Maître de conférences, Didaxis Dipralang 